\title{
The Measurement of the Light Deflection from Jupiter: Experimental Results
}

\author{
E. B. Fomalont \\ National Radio Astronomy Observatory, Charlottesville, VA 22903 \\ efomalon@nrao.edu \\ S. M. Kopeikin \\ Dept. of Physics and Astronomy, University of Missouri-Columbia, Columbia, MO 65211 \\ kopeikins@missouri.edu
}

\begin{abstract}
We have determined the relativistic light deflection of the quasar J0842+1835 as Jupiter passed within $3.7^{\prime}$ on 2002 September 8, by measuring the time delay using the VLBA and Effelsberg radio telescopes at $8.4 \mathrm{GHz}$. At closest approach, General Relativity (GR) predicts a radial (static) deflection of $1190 \mu \mathrm{arcsec}$, and a tangential (retarded) deflection in the direction of Jupiter's motion of $51 \mu$ arcsec. Our experiment achieved an rms position error of $\leq 10 \mu \operatorname{arcsec}$, and measured this retarded deflection to be $0.98 \pm 0.19$ (rms error) times that predicted by GR. The increased positional accuracy for this VLBI phase referencing experiment was achieved by using two calibrator sources. Comments on the interpretation of this experiment, are given.
\end{abstract}

Subject headings: gravitation - quasars: individual (QSO J0842+1835) — relativity techniques: interferometric

\section{The Gravitational Deflection of Radio Waves by Jupiter}

Einstein solved the equation of light propagation in the field of a static body, and predicted the deflection of light at the limb of the Sun of $1.75^{\prime \prime}$ (Einstein 1916), and this relativistic deflection was measured for the first time in 1919 (Dyson, Eddington \& Davidson 1920). The deflection prediction by Einstein is equivalent to measuring the time delay of light traveling in a static gravitation field (Shapiro 1964), and was confirmed in a series of experiments, the most precise of which was made with Very Long Baseline Interferometry (VLBI) (Lebach et al. 1995; Eubanks et al. 1997). Kopeikin $(2001,2003)$ generalized the problem of light propagation in the gravitational field of arbitrary moving bodies, and showed that the Lorentz-invariant expression for the relativistic time delay of light (and light deflection), to all orders in $v / c$, depends on the retarded positions of the moving bodies as defined by the retarded Liénard-Wiechert solution of the Einstein gravitational field equations. For Jupiter, as an example, the Lorentz-invariant relativistic time delay between two telescopes (\#1 and \#2), from a source of light at infinity (quasar), is given by the equation (Kopeikin 2001)

$$
\begin{aligned}
\Delta= & \frac{2 G M_{J}}{c^{3}}\left[1+\frac{\mathbf{K} \cdot \mathbf{v}_{J}\left(s_{1}\right)}{c}\right] \\
& \ln \frac{r_{1 J}\left(s_{1}\right)+\mathbf{K} \cdot \mathbf{r}_{1 J}\left(s_{1}\right)}{r_{2 J}\left(s_{2}\right)+\mathbf{K} \cdot \mathbf{r}_{2 J}\left(s_{2}\right)}
\end{aligned}
$$

where $G$ is the gravitational constant, $c$ is the speed of light, $M_{J}$ is the mass of Jupiter, $\mathbf{K}$ is the unit vector from the barycenter of the solar system to the quasar, and $\mathbf{v}_{J}\left(s_{1}\right)$ is the coordinate velocity of Jupiter taken at the retarded time $s_{1}$. The retarded times $s_{1}$ and $s_{2}$ are calculated according to the gravity null-cone equations, as defined 
by the Liénard-Wiechert retarded solution of the Einstein gravity field equations which contain a constant in the wave operator that we denote as $c_{g}$ (Kopeikin 2003; Will 2003). The retarded times are

$$
\begin{aligned}
& s_{1}=t_{1}-\frac{1}{c_{g}}\left|\mathbf{x}_{1}\left(t_{1}\right)-\mathbf{x}_{J}\left(s_{1}\right)\right| \\
& s_{2}=t_{2}-\frac{1}{c_{g}}\left|\mathbf{x}_{2}\left(t_{2}\right)-\mathbf{x}_{J}\left(s_{2}\right)\right|
\end{aligned}
$$

where $t_{1}, t_{2}$ are times of arrival of the radio signal from the quasar to the first and second VLBI stations respectively, having coordinates $\mathbf{x}_{1}\left(t_{1}\right)$ and $\mathbf{x}_{2}\left(t_{2}\right)$, and $\mathbf{x}_{J}(s)$ is coordinate of Jupiter taken at the retarded time. The distances between each telescope and Jupiter are given by $r_{1 J}=\left|\mathbf{r}_{1 J}\right|, r_{2 J}=\left|\mathbf{r}_{2 J}\right|, \mathbf{r}_{1 J}\left(s_{1}\right)=\mathbf{x}_{1}\left(t_{1}\right)-\mathbf{x}_{J}\left(s_{1}\right)$ and $\mathbf{r}_{2 J}\left(s_{2}\right)=\mathbf{x}_{2}\left(t_{2}\right)-\mathbf{x}_{J}\left(s_{2}\right)$, all of which depend implicitly on the value of $c_{g}$. GR predicts that the constant, $c_{g}$, equals the speed of light $c$. If $c_{g} \neq c$, then Eqs. (1) to (3) would not be invariant with respect to the Lorentz transformation. Hence, the experiment measures the numerical value of $c_{g}$ as a test of the Lorentz invariance of the Einstein equations, and is an indirect measurement of the speed of propagation of gravity ${ }^{1}$. Standard data analysis of VLBI observations uses the barycentric time $t$ and the barycentric space coordinates $\mathbf{x}=(x, y, z)$. Hence, all quantities and variables in Eqs. (1)-(3), and hereafter, are expressed specifically in these coordinates. For example, $\mathbf{v}_{J}=d \mathbf{x} / d t$ is the orbital velocity of Jupiter with respect to the barycenter of the solar system, and vector $\mathbf{K}$ defines the direction to the quasar as measured by an observer at rest with respect to the barycenter (see Kopeikin (2001) and Kopeikin \& Fomalont (2002) for more detail).

Relativistic time delay of light in the gravitational field of moving Jupiter was measured in a VLBI experiment on September 8, 2002 when Jupiter passed within $3.7^{\prime}(\sim 14$ jovian radii $)$ of the bright radio quasar $\mathrm{J} 0842+1835$. If Jupiter is at a small angular distance $\theta$ from the quasar, Eq. (1) can be expanded in a Taylor series around the

\footnotetext{
${ }^{1}$ The radio wave from the quasar does not propagate along the gravity null cones (Eqs. (2) and (3)) and, therefore, $c_{g}$ can not be physically identified with the speed of light (Kopeikin 2001, 2003), as was done, for example by Asada (2002)
}

time of arrival ${ }^{2}$. Then, the linearized relativistic time delay, $\triangle\left(\tau_{l, m}\right)$ between two radio telescopes from a VLBI network having numbers $l$ and $m$ and separated by a distance $\mathbf{B}_{l, m}$, can be expressed to sufficient accuracy as

$$
\begin{gathered}
\triangle\left(\tau_{l, m}\right)=-\frac{4 G M_{J}}{c^{3} r_{m J}} \\
{\left[\left(1-\frac{2 \mathbf{n} \cdot \mathbf{v}_{T J}}{c_{g} \theta}\right) \frac{\mathbf{n} \cdot \mathbf{B}_{l, m}}{\theta}+\frac{\mathbf{B}_{l, m} \cdot \mathbf{v}_{T J}}{c_{g} \theta^{2}}\right]}
\end{gathered}
$$

where $\mathbf{v}_{T J}=\mathbf{K} \times\left(\mathbf{v}_{J} \times \mathbf{K}\right)$ is Jupiter's velocity in the plane of the sky, $\mathbf{n}$ is the unit vector from Jupiter to the radio source in the plane of the sky, and $r_{m J}$ is the distances between Jupiter to the $m$ th telescope. The angle $\theta$ is defined by the relationship (Kopeikin (2001), see Eq. (9)), $\cos \theta=$ $-\mathbf{K} \cdot \mathbf{p}$, where $\mathbf{p}=\left(\mathbf{x}_{1}-\mathbf{x}_{J}\left(t_{1}\right)\right) /\left|\mathbf{x}_{1}-\mathbf{x}_{J}\left(t_{1}\right)\right|$, and is calculated from the accurate JPL ephemeris (Standish 2000). The first term in the large brackets is the radial deflection term ${ }^{3}$, and the second term in brackets is the tangential deflection which is in the direction of the velocity of Jupiter with respect to the barycenter of the solar system, $\mathbf{v}_{T J}$, in the plane of the sky. The velocity-dependent terms in Eq. (4) result from the Taylor expansion around the time of arrival of $t_{1}$ of the retarded arguments in Eq. (1). In the analysis of the experimental data, we used Eq. (1)-(3), rather than the approximate form in Eq. (4). This misunderstanding of the analysis process has led to the erroneous claim (Samuel 2003) that no $v / c$ terms are observed with this deflection experiment (see Kopeikin (2003) for more detail).

The constant $c_{g}$, shown explicitly in Eq. (4), is associated in our model of the experiment with a speed of gravity ( scalar and vector modes) propagating along the gravity null-cone Eqs. (2) and (3) in accordance with the physical interpretation of the Liénard-Wiechert solutions of Einstein's equations (Kopeikin 2001, 2003) ${ }^{4}$. We introduce a fit-

\footnotetext{
${ }^{2}$ In the general case when angle $\theta$ is not small the accurate retarded Eqs. (1)-(3) must be used.

${ }^{3}$ This equation treats more precisely the radial deflection than the corresponding equation from (Kopeikin 2001).

${ }^{4}$ The tensor modes (free gravitational waves) also propagate along the gravity null cones but they can not be detected in the near zone of the solar system. Hence, the experiment can not be viewed as a detection of gravitational waves which are too faint to be seen with present VLBI accuracy (Kopeikin et al. 1999).
} 
ting parameter $\delta=c / c_{g}-1$ to measure the difference between the two speeds. For GR, $\delta=0$. Other theoretical models of this experiment (Will 2003; Asada 2002) predict the same time delay given in Eqs. (1) and (4), but only to the first order of $v / c$. However, these different formulations lead to a different interpretation of $c_{g}$. See $\S 4 \mathrm{~b}$ for further discussion.

The order of magnitude of the deflection prediction on September 8 for the $6000-\mathrm{km}$ telescope separation is a delay of 115 psec (deflection of $1190 \mu \operatorname{arcsec})$ for the radial term, and a delay of $4.8 \mathrm{psec}$ (deflection of $51 \mu \mathrm{arcsec}$ ) for the retarded term at the point of closest approach. Although $v_{J} / c \approx 4 \times 10^{-5}$, the additional factor of $1 / \theta$ amplifies the retarded term so that it is $4 \%$ of the radial term ${ }^{5}$. A previous close passage occurred in 1988 March 21 (Treuhaft \& Lowe 1991) and the radial deflection term was measured to an accuracy $\approx 15 \%$ in accordance with GR. With improvements over the years in VLBI techniques, sub-milliarcsecond positional accuracy were now attainable, and measurement of the retarded term was feasible.

\section{The Experimental Strategy}

The VLBI experiment to measure the deflection of light of the quasar J0842+1835 by Jupiter was conducted during five days, centered on September 8, 2002. The radio array consisted of eleven telescopes: ten 25-m diameter telescopes of the Very Long Baseline Array (VLBA), plus the 100$\mathrm{m}$ diameter telescope at Effelsberg, Germany. The observing frequency was $8.45 \mathrm{GHz}$, and ten hours were used for observations on each of 2002 September $4,7,8,9,12$.

An array measures the difference in arrival time, the delay, for a quasar signal to reach each of the telescopes. Using a widely spaced array, extremely high positional accuracy can be obtained. However, this delay is contaminated by many effects, both internal to and external to each telescope system. To remove these effects, radio astronomers use the technique of phase referencing (alternating observations of two sources) whereby observations of one source (the calibrator) are used to determine the delay errors asso-

\footnotetext{
${ }^{5}$ The amplification is due to the time lag between present and retarded positions of Jupiter (Kopeikin 2001).
}

ciated with the target source (Beasley \& Conway 1995). If the switching time and source separation between calibrator and target are less than the temporal/spatial variation scale sizes, the relative position between the two sources can be accurately measured. However, the propagation delay along the quasar paths through the ionosphere and troposphere may be so variable in time and angle, that even fast switching will not completely remove these propagation changes, and relative position accuracies $\leq 30 \mu$ arcsec are difficult to obtain even with VLBI techniques.

After several sets of test observations in early 2002 (Fomalont \& Kopeikin 2002), we decided to sequentially observe J0842+1835 with two known nearby calibrators: J0839+1802, separated $0.82^{\circ}$ in position angle $-132^{\circ}$; and $\mathrm{J} 0854+2006$ (=OJ287), separated by $3.36^{\circ}$ in position angle $63^{\circ}$. The sources are identified as quasars with high redshift, and they lie on a nearly straight line, which was exploited in the calibration procedure. The observing sequence was identical for the 5 observing days: We cycled observations through the three sources in $5.5 \mathrm{~min}$, with about $100 \mathrm{such}$ cycles per day, a reasonable compromise between removing the temporal effects with obtaining good accuracy of the measured delay. This observational technique was an improved variant of that used for the solar bending experiments in 1974 and 1975 (Fomalont \& Sramek 1976). The jovian magnetosphere also produced a radial deflection of the radio waves (directed inward to Jupiter) and this anomalous bending was of concern. However, calculations suggested that this bending would be significantly less than the retarded deflection term, and special observing techniques (observing at two frequencies simultaneously) would have reduced the sensitivity of the experiment to the gravitational bending. The magnetosphere bending is discussed in more detail below.

\section{The Data Reduction}

\subsection{The Radio Interferometer Response}

A radio interferometer measures the complex spatial coherence function of the electromagnetic radiation field $C_{l, m} e^{i 2 \pi \psi_{l, m}}$ at a frequency $\nu$ between two telescopes denoted by $l$ and $m$. If the intercepted electromagnetic radiation field is dominated by a small-diameter radio source, then 
the response of the interferometer, denoted as the complex visibility function $V_{l, m} e^{i 2 \pi \phi_{l, m}}$, is

$$
\begin{array}{r}
V_{l, m} e^{i 2 \pi \phi_{l, m}}=C_{l, m} e^{\left(i 2 \pi \psi_{l, m}\right)} \\
\hat{G}_{l} \hat{G}_{m}^{*} e^{\frac{i 2 \pi \nu}{c}\left(\Delta \tau_{l}-\Delta \tau_{m}\right)}
\end{array}
$$

The complex gains of the telescopes, $\hat{G}_{l}$, contain the amplification and phase shifts that are introduced by each telescope system (* denotes complex conjugate), and the residual time delay (observed - model) of the signal from the radio source to each telescope is denoted by $\triangle \tau_{l}$. For an array of $N$ telescopes, the visibility function is sampled simultaneously with $N(N-1) / 2$ interferometers, 55 pairs for an eleven-element array.

The accurate delay model was calculated using the Goddard Space Flight Center (GSFC) CALC software package version $9.1^{6}$, which uses the most recent parameters associated with the earth rotation and orientation, nutation, the terrestrial reference frame, and the radio source positions defined on the celestial reference frame (Ma et al. 1998). We have slightly modified the CALC package to incorporate the gravitational deflection from all significant solar system bodies including the retardation term, as given by Eq. (1). We have assumed that the PPN parameter $\gamma=1$, because it has been measured to $0.1 \%$ accuracy (Lebach et al. 1995; Eubanks et al. 1997). We could not improve this value in our experiment, and its uncertainty produces a deflection offset $\leq 1 \mu \mathrm{arcsec}$, much less than the experimental accuracy that we achieved. Parameters from which telescope delay variability could be determined were monitored during the observations (egs. ground weather conditions), and ionospheric data was available from GSFC data archives collected by Global Positioning Satellites (GPS).

The phase part of the complex visibility function (called simply the phase) for each source $a$, can be written as

$$
\begin{gathered}
\phi_{l, m}^{a}(t)=\frac{\nu}{c}\left[\mathbf{B}_{l, m} \cdot \triangle \mathbf{K}^{a}(t)+\right. \\
\left.\triangle \mathbf{B}_{l, m}(t) \cdot \mathbf{K}^{a}+\triangle C_{l, m}(t)+\triangle A_{l, m}^{a}(t)\right]+\psi_{l, m}^{a}(t)
\end{gathered}
$$

where $\mathbf{B}_{l, m}$ is the model separation between telescope $l$ and $m, \triangle \mathbf{B}_{l, m}(t)$ is the separation error; $\mathbf{K}^{a}$ is the model position of the ath source,

\footnotetext{
${ }^{6}$ www.sgl.crestech.ca/IVS-Analysis/software_tools /calc_solve/datafiles.htm
}

$\triangle \mathbf{K}^{a}(t)$ is the position offset error for the ath source, $\triangle \mathrm{C}_{l, m}(t)$ is the residual clock delay between telescopes, and $\triangle A_{l, m}^{a}(t)$ is the residual tropospheric/ionospheric delay in the direction to the ath source. The structure of the source is given by the phase part of the complex spatial coherence function, $\psi_{l, m}^{a}(t)$. For this experiment, $a$ has three values: $a=0$ (J0842+1835), $a=1(\mathrm{~J} 0839+1802)$, $a=2$ (J0854+2006). The occasional lobe ambiguities (arbitrary turns of phase) of the phase measurements were easily determined because of the accuracy of the delay model.

\subsection{The Source Structure}

The accuracy of the experiment depends in part on the stability of the three sources between September 4 to 12. In terms of variables in Eq. (6), the intrinsic change of position, $\triangle \mathbf{K}^{a}(t)$, of each source, and the visibility structure phases variations, $\psi_{l, m}^{a}(t)$, must be small. The structures for each source on each day were determined from their respective complex visibility function using self-calibration techniques for radio imaging (Walker 1995). This imaging/deconvolution iteration process does not determine the accurate positions of each source, only its shape. The derived images of the three quasars during the experiment are shown in Fig. 1. All three sources show the typical structure associated with most luminous radio sources, a bright component (core) at one end of the structure containing an appreciable part of the emission, and more extended emission, often with a secondary bright component. There were no apparent changes in the intensity and shape of the sources, except for small changes in J0854+2006 at the 1\% contour level. These properties are in agreement with previous observations of J0842 (sources names will be abbreviated) and J0839 which show little long term structure changes (Fey \& Charlot 2000; Beasley et al. 2001), whereas J0854 is a known variable source.

The observation dates are symmetric with respect to September 8. Hence, even if there are long-term small structure changes over the experiment, the deviation of the average structure phase of each source, based on observations averaged on September 4,7,9 and 12, should be equal to first order to that on September 8 (this averaging is discussed below). Nevertheless, the change in the 
measured phase associated with the structure difference in J0854 between September 4 and 12 is less than 0.003 turn for any baseline, and this corresponds to an effective positional error of less than $3 \mu$ arcsec, even without the above averaging which was incorporated in the reductions. Another indication of very little change over the 7-day duration of the observations is that the separation of the radio core and the secondary component for all sources was stable to $\leq 20 \mu$ arcsec, an accuracy consistent with the sensitivity of the observations and the somewhat diffuse nature of the secondary peak.

The large distance to the radio sources guarantees that any proper motion will be much less than one microarcsec over the week period of the experiment. However, some compact components $(<50 \mu$ arcsec $)$ in radio sources vary slightly in intensity over hour time-scales because of galactic scattering at a distance of $\approx 300 \mathrm{pc}$ (Bignall et al. 2003). It is possible that such scattering also irregularly moves the apparent position of the component. However, we detected no short-term intensity variations in any source greater than $4 \%$ over periods of a few hours, and concluded that the compact components are not affected by interstellar scattering. However, we emphasize that such movement of the source position, if it existed, could be detected in this experiment as larger than expected residual phases with time.

\subsection{Removing the Temporal and Spatial Phase Errors}

With the removal of the source structure terms (we used the average source structure over the five observing days), the phase in Eq. (6) becomes circular among the telescopes; that is $\phi_{l, m}=$ $\phi_{l, n}+\phi_{n, m}$, apart from stochastic noise. Therefore, there are only 10 independent phases for an array of 11-telescopes. We can thus choose a reference telescope, $M$, and write Eq. (6) for each source $a$ and telescope $l$, at time $t_{a}$ as

$$
\begin{array}{r}
\phi_{l, M}^{a}\left(t_{a}\right)=f_{l, M}\left(t_{a}\right)+ \\
\mathbf{g}_{l, M}\left(t_{a}, \mathbf{K}^{a}\right)+\triangle \mathbf{K}^{a}\left(t_{a}\right) \cdot \mathbf{B}_{l, M}
\end{array}
$$

where $f_{l, M}\left(t_{a}\right)$ is a function of time, which is dominated by the clock drifts and other instrumental variations at each telescope, and is independent of the source position. The term $\mathbf{g}_{l, M}\left(t_{a}, \mathbf{K}^{\mathbf{a}}\right)$ describes the temporal and spatial properties of the delay screen above each telescope near the position of the three sources. The last term in Eq. (7) cannot be written as part of the first two terms since it depends on an unknown, but small, offset for each source from the assumed value. We used Mauna Kea (MK), HI as the reference telescope for most of the observations since this telescope is at a high site with generally good atmospheric conditions. For the first three hours of each day when the source elevation at MK was $<20^{\circ}$, we used the Los Alamos, NM telescope as the reference telescope.

The properties of the several components of delay which contribute to the time variable phase screen over each telescope are well-known. The phase error associated with the uncertain location of a telescope $\triangle \mathbf{B}_{l, M}$, which includes any deviation of the earth rotation and orientation and nutation terms from those used in correlator CALC Model, are smooth functions of time and direction in the sky. The component produced by the changing refractivity of the troposphere and ionosphere toward the sources from relatively large clouds and diurnal changes persists for tens of minutes and are generally larger than $5^{\circ}$ in angular size. On the other hand, small tropospheric clouds and ionospheric events produce quasi-stochastic changes above each telescope. In their extreme behavior, these rapidly changing delays have time scales less than a few minutes and degrees, making astrometric VLBI observations useless.

We can expand the first two terms in Eq. (7) into a Taylor series around $\left(t_{a}-t_{0}\right)$ and $\left(\mathbf{K}^{a}-\mathbf{K}^{0}\right)$. Since J0842 and the two calibrators lie nearly linearly in the sky, the angular part of the expansion is only needed in the direction of the source alignment in the $\mathrm{sky}^{7}$. The first order terms can be estimated by a sum of the observed calibrator phases, weighted by their separation from J0842 in the sky, and in time. Thus, the calibrated phase for J0842, $\Phi\left(t_{0}\right)_{l, M}$ at its observation time, $t_{0}$, for any day for telescope $l$ becomes

$$
\begin{array}{r}
\Phi\left(t_{0}\right)_{l, M}=\phi^{0}\left(t_{0}\right)_{l, M}-\left(0.80 \phi^{1}\left(t_{0}\right)_{l, M}+0.20 \phi^{2}\left(t_{0}\right)_{l, M}\right) \\
\approx \mathbf{B}_{l, M} \cdot\left(\triangle \mathbf{K}^{0}\left(t_{0}\right)-0.80 \triangle \mathbf{K}^{1}\left(t_{0}\right)-0.20 \triangle \mathbf{K}^{2}\left(t_{0}\right)\right)
\end{array}
$$

where $\phi^{1}\left(t_{0}\right)$ and $\phi^{2}\left(t_{0}\right)$ are the phases of sources 1 or 2 , each measured at times $t_{1}$ and $t_{2}$, but

\footnotetext{
${ }^{7}$ Three calibrator sources are needed in the general case.
} 
linearly-interpolated to time $t_{0}$. In other words, the algorithm in Eq. (8) defines how the measured phases for J0839 and J0854 are combined to remove, to first order, the temporal and angular phase change in the sky at the position of J0842 at the time of its observation at $t_{0}$. The calibrated phase of J0842, thus, depends explicitly on the change of position of the three radio sources with time. The short time-scale and small angular-scale fluctuations are not removed, but these are nearly stochastic and average out over longer periods of time. They add to the uncertainty of the results, but do not contribute to any systematic biases. Second order terms, which are not removed from the interpolation used in Eq. (8), have been minimized by using precise apriori parameters available from the CALC package and available tropospheric and ionospheric data associated with each telescope from the GPS data base.

Examples of the effectiveness of this calibration process are shown in Fig. 2 for the Owens Valley, CA to Mauna Kea, HI baseline on September 9. The observed phases, $\phi^{a}(t)$, (top plot) for the three sources follow each other over the day, but are separated at any time by an amount which is varying, caused by a phase wedge in the atmosphere. The displacement of phase between J0854 and J0842 is larger and in the opposite sense than that from J0839 and J0842, as expected from linear phase wedge. The calibrated phase $\Phi$ of J0842, (bottom) is relatively smooth and near zero phase. The relatively small scatter is caused by fluctuations of phase shorter in temporal scale than about five minutes, or smaller in angular scale than five degrees. The signal to noise inherent in the observations cause only a small part of the scatter.

There are periods of time when the phase stability shown by all three sources noticeably deteriorates, and the calibrated phase of J0842 becomes large. These periods often occur when the source is at a low elevation, typically less than $20^{\circ}$, when large and non-linear tropospheric and ionospheric phase are more likely. Occasionally at high elevations during periods of rainy and windy conditions, VLBI observations are useless. These periods of obvious poor phase stability were removed from further consideration, and consisted of $15 \%$ of the original data. On the average the phase stability at sites with dry conditions was more stable than at those with humid conditions.
The calibrated phases, $\Phi_{l, M}(t)$, were then averaged for each day over a one hour period, with an estimated rms error determined from the scatter, which is about 0.02 turns per point. In Fig. 3 , we show these averaged calibrated phases for two telescopes. The calibrated phases repeat extremely well from day to day to an rms scatter of about 0.02 turns. Even a slight negative offset at $\mathrm{GST} \approx 15 \mathrm{hr}$ repeats, and is produced by $\mathrm{a} \sim 50 \mu \operatorname{arcsec}$ offset of the source position from that assumed in the model, as given in Eq. (8).

\subsection{The Deflection on September 8}

The difference between the calibrated phase on September 8, and the average of the phases from the other days, is shown in Fig. 4 for the same two telescopes as in Fig. 3. Assuming that the position of all three sources remain fixed with respect to the apriori model, which includes the radial and retarded gravitational bending predicted by GR, the phases in Fig. 4 should scatter around zero phase, corresponding to $\delta=0$. The curve given by $\delta=-1$ shows the expected calibrated phase on September 8 if the retarded deflection term were zero. Fig. 4 already demonstrates that $\delta \approx 0$, and that the experiment has clearly detected the retarded component of the light deflection.

An analysis of the effect of the jovian magnetosphere, has been described fully (Kopeikin \& Fomalont 2002), and is summarized here. The Galileo spacecraft provided an estimate of the average electron plasma density, $N_{0}$, near the jovian surface. We assumed a spherically-shaped magnetosphere with a plasma density which decreases as $N_{0}\left(R_{J} / r\right)^{2+A}$ (where $R_{J}=7.1 \times 10^{7} \mathrm{~m}$ is the mean radius of Jupiter and A is an exponent reflecting different magnetospheric models), and integrated the estimated plasma delay at $8.45 \mathrm{GHz}$ along the propagation path of J0842 at closest approach $\left(3.7^{\prime}\right.$ or $\left.13 R_{J}\right)$. We obtain magnetospheric deflection estimates of $17.5,1.0,0.02 \mu \operatorname{arcsec}$ for $A=0.0,1.0,2.0$, respectively. Based on the analogy with radial distribution of the solar corona, which suggests $A \approx 0.33$, we have determined the position shift associated with this jovian magnetosphere model (it is in the opposite direction of the gravitational radial deflection of light), and we have shown its estimated phase contribution by the dashed line in Fig. 4. This estimate is probably substantially larger than the actual value on 
this day.

There are two ways in which we can combine the data in order to determine the mean gravitational deflection of J0842. First, we averaged the data for each telescope, and determined $\delta$ associated with each telescope, and these results are shown in Table 1, listed in decreasing order of accuracy. We find that the most accurate determinations are associated with telescopes in the southwest USA, about $5000 \mathrm{~km}$ from MK, because they are located at places with a relatively stable and dry atmosphere. Telescopes in more humid locations give poorer results. The Effelsberg telescope, however, was critical in the determination of the accurate structure of the radio sources. All telescope determinations are consistent with $\delta=0$ when their estimated errors are considered.

Another method of display is to produce an image from the calibrated phase data for all baselines, which are measured during any one-hour period. The location of the peak of the image is the position of J0842, and these positions are shown in Fig. 5. The east/west position of the source is more accurately determined than the north/south position since the array spans mostly in the east/west direction. The east/west positions clearly show that the retarded component of the light deflection has been detected, and is consistent with GR. The red line shows the estimate of a reasonable jovian magnetospheric refraction, and is much smaller than the retarded deflection, particularly in the east/west direction.

The weighted average value of the data for the observations over September 8, regardless of how the data are averaged (by telescope or by time), is

$$
\delta=-0.02 \pm 0.19 \quad 1-\sigma \text { error }
$$

Hence, the measured retarded deflection associated with the retarded position of Jupiter given by Eqs. (1)-(3), is in good agreement with the prediction of GR. Our interpretation of $\delta$ sets a bound of $c_{g}=(1.06 \pm 0.21) c($ see $\S 4 . \mathrm{b})$

\section{Discussion}

\subsection{The Measurement Results}

The measurement of the retarded deflection component in Eq. (1) is an accurate experimental confirmation of General Relativity in the solar system, using the motion of Jupiter. To determine $\delta$ with sufficient accuracy, improvements in the technique of phase-reference VLBI were developed.

The use of two calibrators, on either side of J0842, improved the positional accuracy of this phase-referencing VLBI experiment by a factor of more than three compared with previous observations of this type. Because of the relative simplicity of the experimental concept as a classical deflection-type experiment, the uniform observational procedure over the entire experiment, the data redundancy provided by the number of telescopes and the number of days, we are convinced that the result and error estimate are accurate and demonstrated from the Fig. 4 and Fig. 5 which display a significant part of the data. The refraction from the jovian magnetosphere is at least as small as we have estimated (Kopeikin \& Fomalont 2002). Any position jitter caused by interstellar scattering is not significant since the scatter in Fig. 5 around the expected retarded position is no larger than that expected from the errors derived from the scatter in the observations among all of the days.

The two-calibrator technique, developed in this experiment to remove the delay variations of the tropospherically-induced phase wedge above the telescopes, can be used for determinations of the proper motion and parallax of radio sources. With the relative positional sensitivity of $10 \mu \mathrm{arcsec}$, the trigonometric parallax of stars and pulsars as far as $10 \mathrm{kpc}$ can be determined; spacecraft can be tracked with an accuracy of $30 \mathrm{~m}$ near Jupiter; and the proper motion of radio sources in M31 can be determined to $5 \mathrm{~km} / \mathrm{sec}(1 \mu \mathrm{arcsec} / \mathrm{yr})$ over ten years. However, the significant structure changes in many calibrator sources over periods longer than a few months may dominate the error budget at angular levels of $>50 \mu$ arcsec for the long-term astrometric projects. Expected improvements in the accuracy of the celestial frame over the next decade may permit the determination of calibrator core positions and changes needed to reach $10 \mu \operatorname{arcsec}$ accuracy (Ma et al. 1998; Jacobs et al. 2002)

\subsection{The Measurement Interpretation}

Our interpretation of this novel deflection experiment comes directly from the formulation of 
Kopeikin $(2001,2003)$ where the retarded deflection term (and the availability of the September 8, 2002 Jupiter experiment) was initially proposed. The interpretation is that the position of Jupiter at the VLBI delay given by Eq. (1) must be taken at the retarded time with respect to the time of observation, as defined by the solution of the gravity null cone Eqs. (2) and (3). This retardation is due to the finite speed of gravity and can be observed. The measured retarded deflection, as expressed by the parameter $\delta$, is in agreement with GR to $20 \%$ accuracy, and that the formulation, summarized by Eqs. (1)-(3), is confirmed to the first order in $v / c$. If $\delta \neq 0$, then the constant $c_{g}$ in the wave operator of the Einstein equations is not equal to the speed of light numerically, and GR (the gravity null cone) would not be invariant with respect to the Lorentz transformation.

Another interpretation has been proposed by Will (2003) who made use of a matching technique that was first employed for solving the same problem by Klioner \& Kopeikin (1992). This formulation assumes that the position of Jupiter in Eq. (1) must be taken at the time of the closest approach of light to Jupiter. Will's formulation is not Lorentz-invariant but approximates the Lorentz-invariant formulation of the time delay up to linearized terms of order $v / c$.

Both formulations ${ }^{8}$ give identical predictions for the deflection of light by a moving body to first order in $(v / c)$. However, the physical meaning attached to the measurement is quite different. The Kopeikin (2001) interpretation is related to the Lorentz-invariant definition of the gravity null cone and the finite speed of propagation of gravity as predicted by the Liénard-Wiechert solution of Einstein's equations. Kopeikin (2003) also associates the $v / c$ effects with the gravitomagnetic dragging of the light ray caused by the translational motion of Jupiter with respect to the barycenter of the solar system. The Will (2003) and Asada (2002) interpretation is that the $v / c$ term is related to the aberration of light that

\footnotetext{
${ }^{8}$ We suggest that the reader compare the treatment of the September 8, 2002 experiment by Kopeikin (2001, 2003), by Will (2003), by Asada (2002), for a fuller understanding of the different interpretations and mathematical techniques involved in derivation of the basic equations. Papers by Kopeikin \& Schäfer (1999); Klioner \& Kopeikin (1992); Klioner (2003); Carlip (2000) may be valuable as well.
}

diverges from our interpretation at terms of order $v^{2} / c^{2}$. Our measurement, unfortunately, is not sufficiently accurate to detect terms of order $v^{2} / c^{2}$ which can distinguish between the existing interpretations experimentally. This could be a challenge for the next generation of space VLBI or other astrometric space missions like SIM or GAIA. Relativistic deflection terms of order $v^{2} / c^{2}$ for light grazing Jupiter's limb, may reach a magnitude of $1 \mu \mathrm{arcsec}$ and can be, in principle, observable.

What is clear is that if this experiment had measured $\delta$ significantly different from zero, then a problem would exist with the current formulation of General Relativity and, as such, any interpretation of the result would be ambiguous and depend on the theoretical generalization of the Einstein equations that was chosen. However, this measurement of the deflection of light by Jupiter is in good agreement with General Relativity and demonstrates experimentally that the Einstein Equations are Lorentz-invariant and the retarded position of Jupiter in Eq. (1) should be used in the determination of the deflection of light in accordance with Eqs. (2) and (3).

The National Radio Astronomy Observatory is a facility of the National Science Foundation, operated under cooperative agreement by Associated Universities, Inc. We thank the support from the Department of Physics and Astronomy, College of Arts and Science, and the Research Council of the University of Missouri-Columbia. The Max-Planck-Institut für Radioastronomie operates the Effelsberg 100-m Radio Telescope. We thank the VLBA staff for their help and support in obtaining and correlating the data. Support from the Eppley Foundation for Research (award \#002672) and the Technical Research Council of Turkey (TUBITAK) is greatly appreciated. SK acknowledges the Department of Physics of the Istanbul Technical University for their hospitality. We thank Norbert Bartel and two anonymous referees for their comments and suggestions.

\section{REFERENCES}

Asada, H. 2002, ApJ, 574, L69

Beasley, A. J., Gordon, A. J., Peck, A. B., Petrov, 
L., MacMillan, D. S., Fomalont, E. B. \& Ma, C. 2002, ApJ, 141. 13

Bignall, H. E., Jauncey, D. L., Lovell, J. E .J., Tzioumis, A. K., Kedziora-Chudczer, L., Macquart, J.-P, Tingay, S. J., Rayner, D. P. \& Clay, R. W. 2003, ApJ, 585, 653

Beasley, A.J. \& Conway, J.E. 1995, in ASP Conf. Ser. 82, Very Long Baseline Interferometry and the VLBA, ed. J.A. Zensus, P.J. Diamond \& P.J. Napier (San Francisco: ASP) 328

Carlip, S. 2000, Phys. Lett. A, 267, 81

Dyson, F. W., Eddington, A. S. \& Davidson, C. 1920, Phil. Trans. Roy. Soc. (London), 220A, 291

Fey, A.L \& Charlot, P. 2000, Astrophys. J. Suppl, 128,17

Einstein, A. 1916, Ann. d. Phys., 49, 769

Eubanks, T. M., Matsakis, D. N., Martin, J. O., Archinal, B. A., McCarthy, D. D., Klioner, S. A., Shapiro, S., \& Shapiro, I. I. 1997, American Physical Society, APS/AAPT Joint Meeting, April 18-21, 1997, abstract \#K11.05, 1105

Fomalont, E.B. \& Kopeikin, S. M. 2002 in: Proc. 6th European VLBI Network Symp., eds. E. Ros, R.W. Porcas, A.P. Lobanov \& J.A. Zensus, (Bonn: MPIfR) 53

Fomalont, E.B. \& Sramek, R.A. 1975, Phys. Rev. Lett. 36, 1475

Klioner, S.A. \& Kopeikin, S.M. 1992, Astron. J., 104, 897

Klioner, S.A. 2003, Astron 83 Astroph., 404, 783

Kopeikin, S.M. 2001, Astrophys. J. , 556, L1

Kopeikin, S.M. 2003, Phys. Lett. A, 312, 147

Kopeikin, S.M. and Fomalont, E.B. 2002, in: Proc. 6th European VLBI Network Symp., eds. E. Ros, R.W. Porcas, A.P. Lobanov \& J.A. Zensus, (Bonn: MPIfR) 49

Kopeikin, S.M. \& Fomalont, E.B. 2003, Phys. Rev. Let. Comment, submitted

Kopeikin, S. M. \& Schäfer, G. 1999, Phys. Rev. D, 60,124002
Kopeikin, S. M., Gwinn, C. R., Schäfer, G. \& Eubanks, T. M. 1999, Phys. Rev. D, 59, 84023

Jacobs, C. S., et al, 2002, AAS Meeting 201, \#76.10

Lebach, D.E., Corey, B.E., Shapiro. I.I., Ratner, M.I., Webber, J.C., Rogers, A.E.E., Davis, J.L. and Herring, T.A. 1995, Phys. Rev. Lett., 75, 1439

Ma, C., Arias, E.F., Eubanks, T.M., Fey, A.L., Gontier, A.-M., Jacobs, C.S., Sovers, O.J., Archinal, B.A., Charlot, P. 1998, Astron. J., 116,516

Samuel, S. 2003, Phys. Rev. Lett, 90, 231101

Shapiro, I. I. 1964, Phys. Rev. Lett., 13, 789

Standish, M. 2000, IAU Colloq. 180: Towards Models and Constatns for Sub-Microarcsecond Astrometry, 120

Treuhaft, R. N. \& Lowe, S. T. 1991, Astron. J, 102,1879

Walker, R. C. 1995, in ASP Conf. Ser. 82, Very Long Baseline Interferometry and the VLBA, ed. J.A. Zensus, P.J. Diamond \& P.J. Napier (San Francisco: ASP) 247

Will, C. M. 1993, Theory and Experiment in Gravitational Physics (Cambridge: Cambridge University Press

Will, C. M. 2003, ApJ, 590, 683

This 2-column preprint was prepared with the AAS LATEX macros v5.0. 
TABLE 1

Telescope Solutions For $\delta$

\begin{tabular}{lcc}
\hline \hline \multicolumn{1}{c}{ Telescope } & $\begin{array}{c}\text { Baseline to MK } \\
(\mathrm{km})\end{array}$ & $\delta$ \\
& & \\
\hline Hancock, NH & 7500 & $-0.02 \pm 0.25$ \\
Los Alamos, NM & 4970 & $-0.20 \pm 0.34$ \\
Kitt Peak, AZ & 4490 & $+0.52 \pm 0.38$ \\
Owens Valley, CA & 4015 & $-0.45 \pm 0.42$ \\
Pie Town, NM & 4800 & $+0.30 \pm 0.44$ \\
Fort Davis, TX & 5130 & $-0.09 \pm 0.52$ \\
North Liberty, IA & 6160 & $-0.37 \pm 0.53$ \\
Brewster, WA & 4400 & $+0.14 \pm 0.62$ \\
Saint Croix, VI & 8610 & $-0.82 \pm 0.85$ \\
Effelsberg, Germany & 10300 & $+1.94 \pm 1.60$ \\
$<\delta>$ & & $-\mathbf{0 . 0 2} \pm \mathbf{0 . 1 9}$ \\
\hline
\end{tabular}



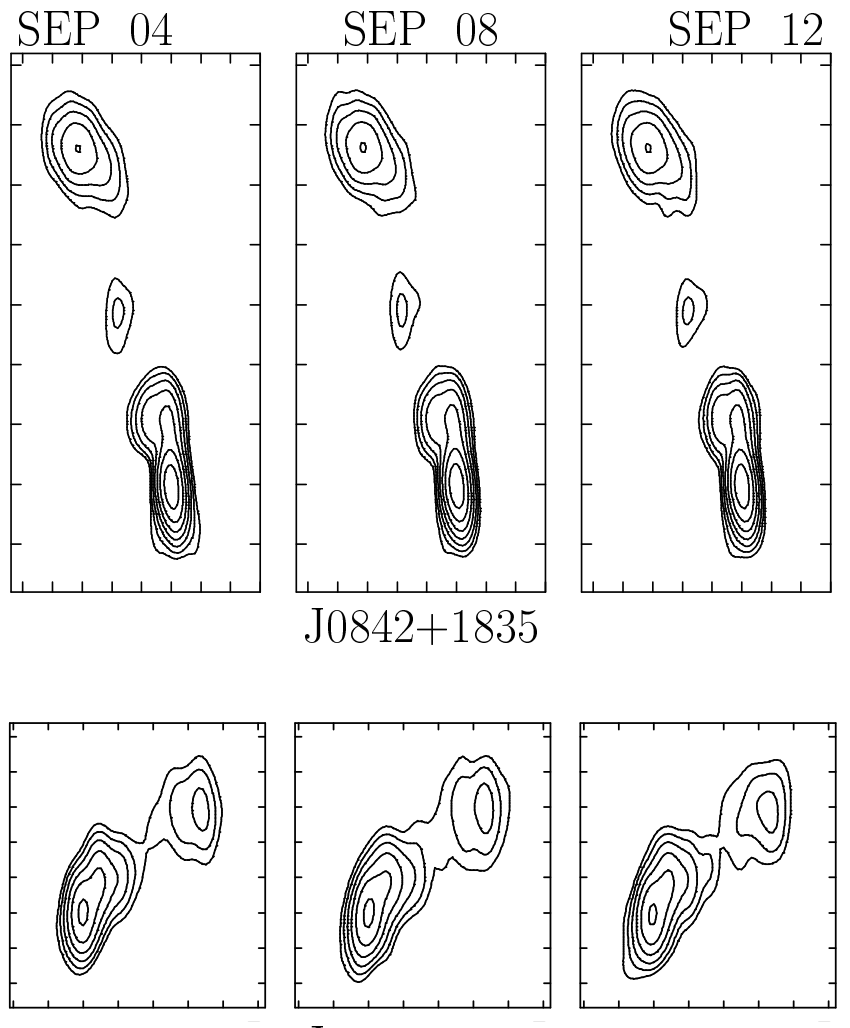

J0839+1802
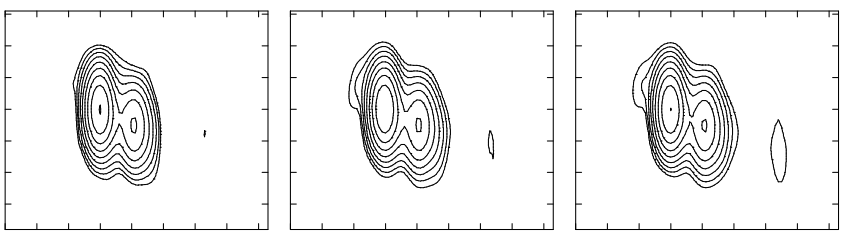

J0854+2006

Fig. 1 - The Structure of the Three Quasars during the Experiment: The contour images for the three quasars on September 4, 8 and 12, are shown from left to right. North is up and east is to the left. The first row shows J0842+1835 with a lowest contour of $3.0 \mathrm{mJy} /$ beam, about $1 \%$ of the peak brightnesses of 306.4, 308.5, 308.4 $\mathrm{mJy} /$ beam from left to right. The second row shows J0839+1802 with a lowest contour of $2.0 \mathrm{mJy} /$ beam, about $2.8 \%$ of the peak brightnesses of $74.6,75.5,70.1 \mathrm{mJy} / \mathrm{beam}$. The third row shows J0854+2006 with a lowest contour of $7.0 \mathrm{mJy}$, about $0.4 \%$ of the peak brightnesses of 1834, 1778, $1799 \mathrm{mJy} /$ beam. The contour levels increase by a factor of two in brightness. The source J0854+2006 shows the emergence of a faint component to the east and a brightening of a weak component to the west. The resolution is $1.5 \times 1.0$ mas (full-width at half-maximum of an elliptical Gaussian beam), and the tick marks are separated by 1 mas (except 2 mas for the N/S axis of J0842+1835), and the lowest contour levels are about 5 times the rms noise. 


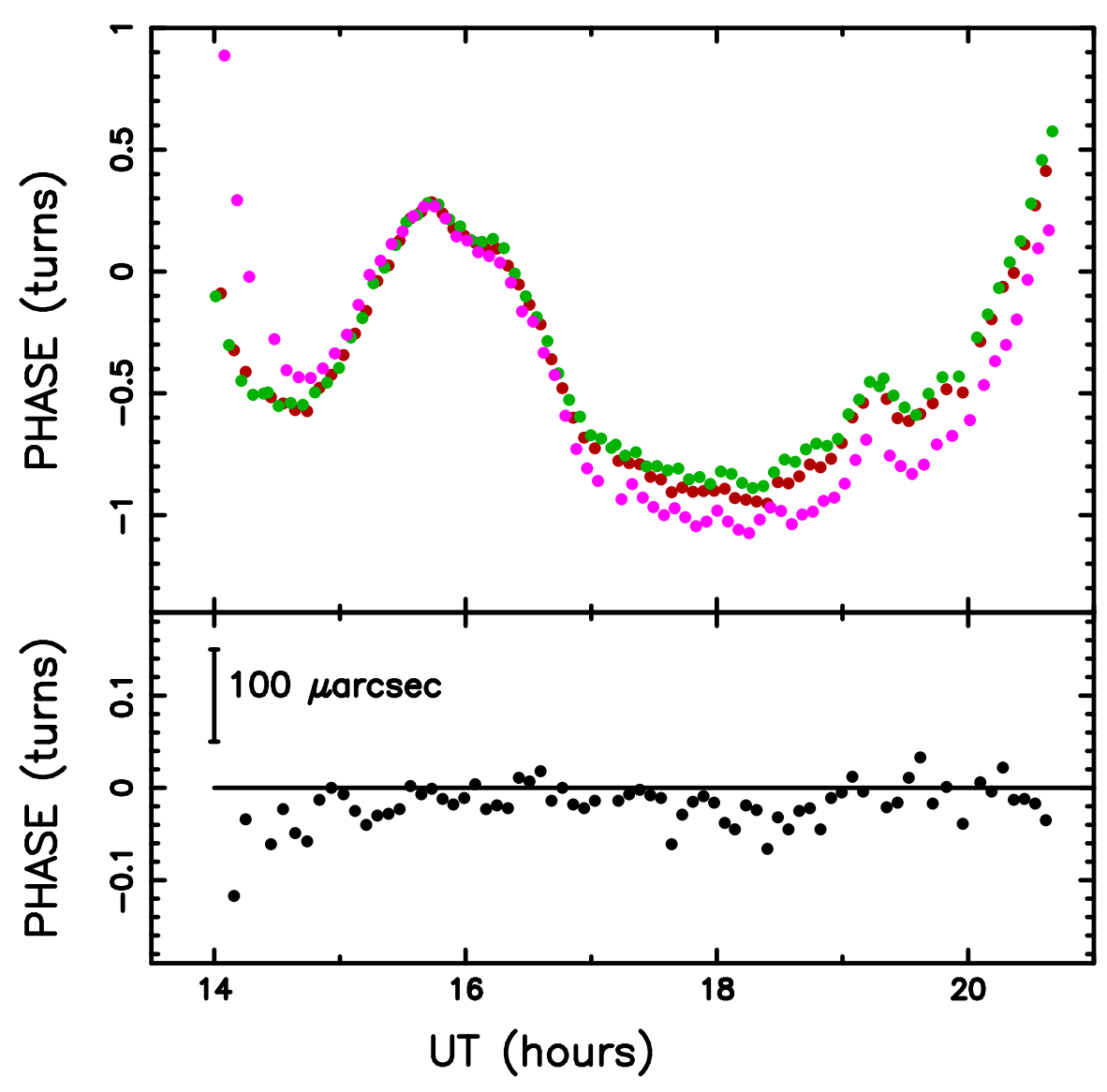

Fig. 2 - Removing the Residual Tropospheric and Ionospheric Delay Variations: The top plot shows the measured phase for the Owens Valley, CA, to the Mauna Kea, HI baseline on September 9 for each source. The red points are for J0842, the green points for J0839 and the purple points for J0854. The bottom plot shows the corrected phase for J0842 after using the linear interpolation of J0839 and J0854, defined by Eq. (7). The large phase gradient between UT $14 \mathrm{~h}$ and $15 \mathrm{~h}$ is produced by the low elevation of the sources, and these data have been removed from further consideration. 


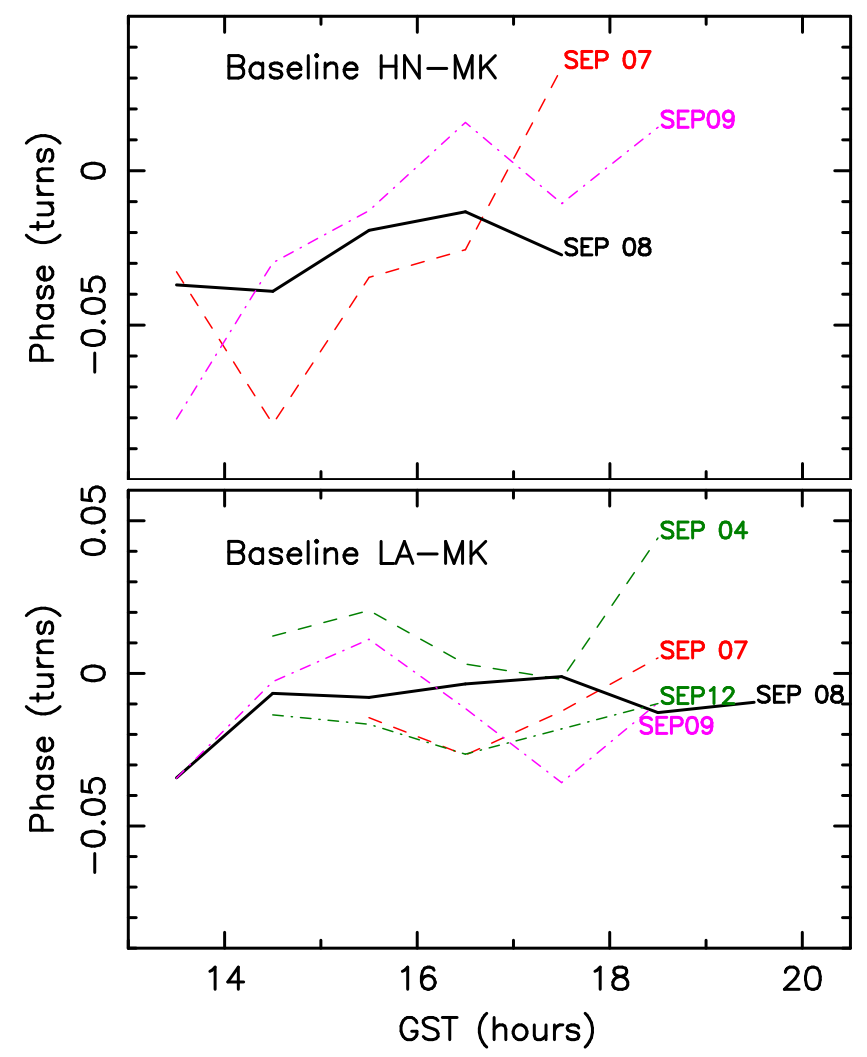

Fig. 3 - The Calibrated Phases for the HN-MK and the LA-MK baselines for all Days. The calibrated phases, averaged to one hour intervals on each day, for the Hancock, NH (top) and Los Alamos, NM (bottom) baselines to Mauna Kea, HI are connected by the line segments, with the day indicated at the right end of each line. The September 8 line is black. The error bars (not shown) are typically 0.02 turns per point. The Hancock telescope was not in use on Sep 4 and 12 because of mechanical problems. 


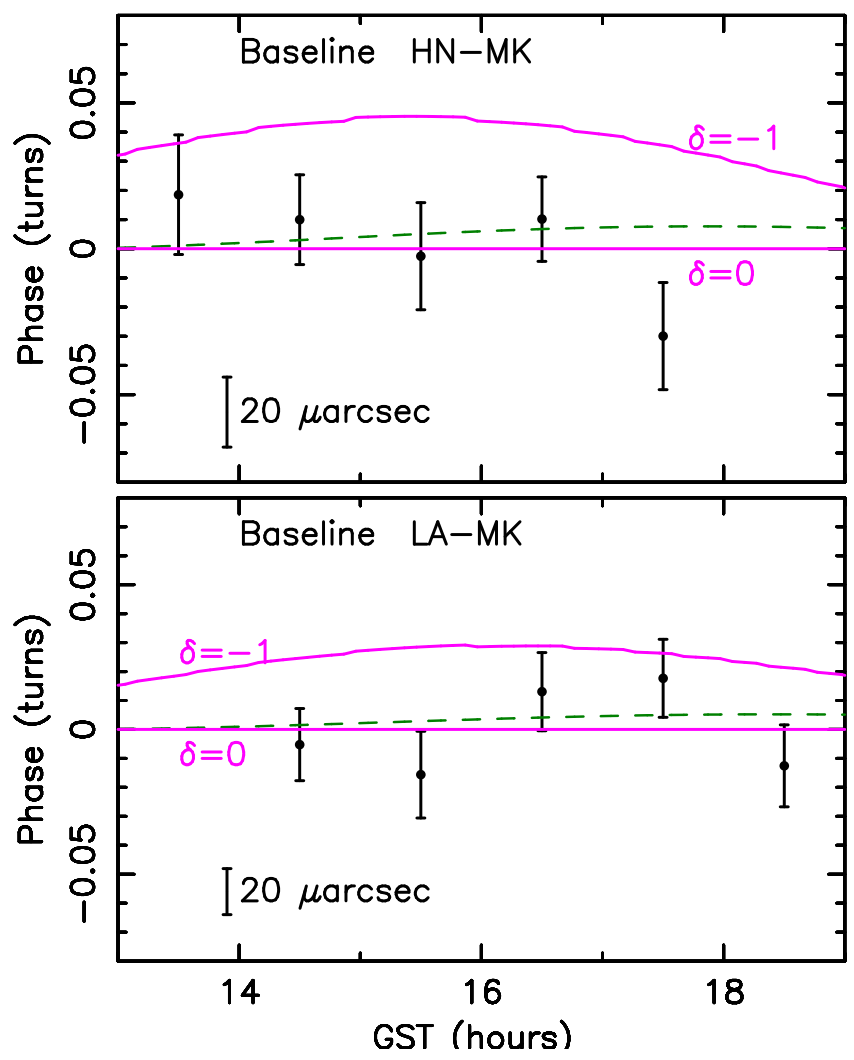

Fig. 4 - The Differential Calibrated Phase for September 8: The phase difference between the calibrated phase on September 8 , and the average of that on the other days are given for Hancock (top) and Los Alamos (bottom) telescopes to Mauna Kea. The errors are derived from the scatter among the off-Jupiter days and the estimated error per point on September 8. The blue curve for $\delta=0$ is that expected from GR; the pink curve for $\delta=-1$ is that expected for no retarded deflection component. The magnetosphere refraction estimate (see text) is shown by the dashed green line. 

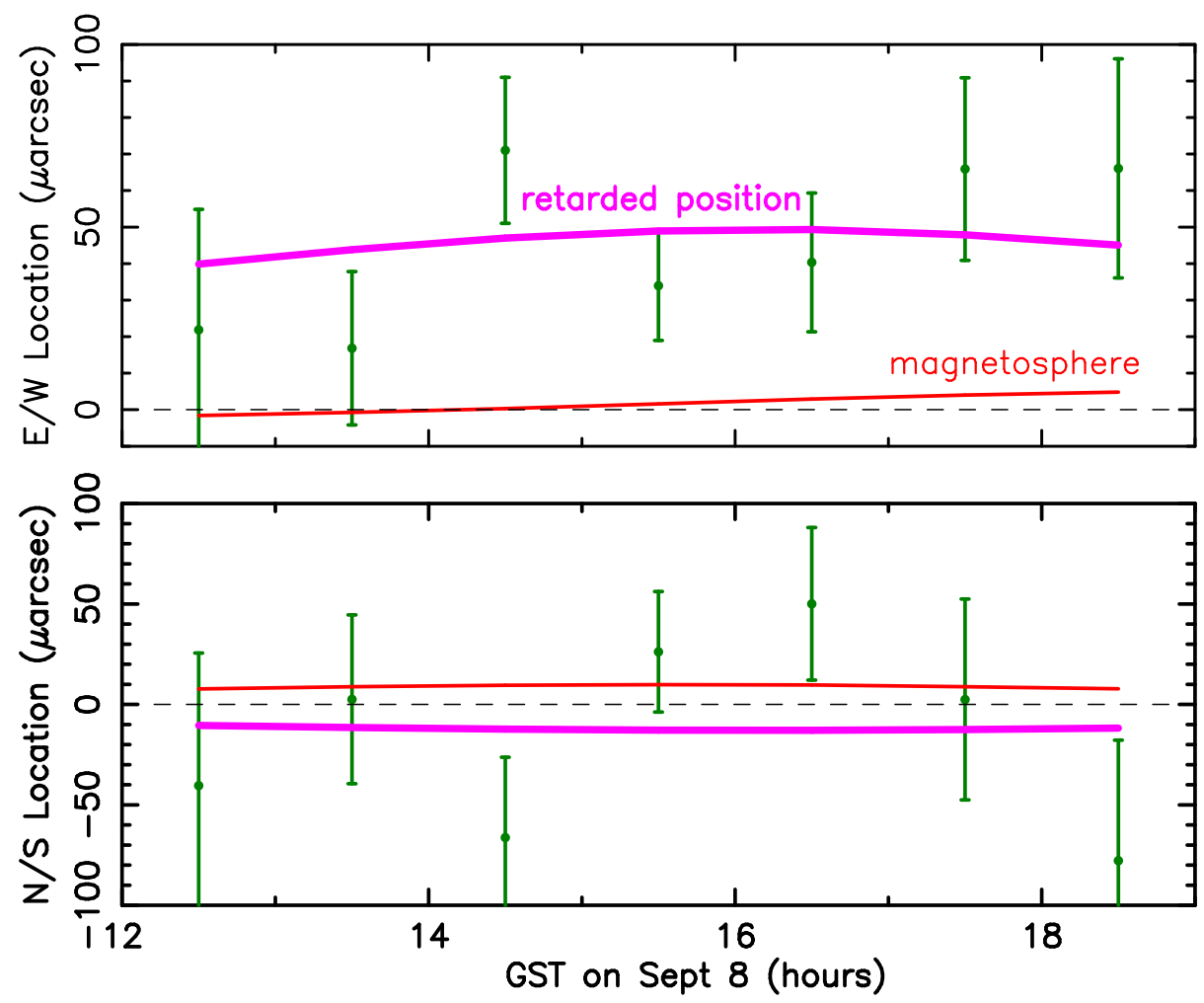

Fig. 5 - The Retarded Deflection of J0842+1835 on September 8, 2002: The plotted points and 1- $\sigma$ error estimates show the retarded position every hour for J0842+1835; that is, the measured deflection in the direction of Jupiter's motion. The radial deflection term of $\approx 1200 \mu \operatorname{arcsec}$ has been removed. The top plot shows the east/west position and the bottom plot shows the north/south position. The expected retarded deflection from GR $(\delta=0)$ is shown by the thick purple line. The estimated jovian magnetosphere refraction is shown by the thin orange line. 